\title{
Geriatric Nutritional Risk Index Predicts Prognosis in Hepatocellular Carcinoma After Hepatectomy: A Propensity Score Matching Analysis
}

Hiroki Kanno ( $\sim$ kanno_hiroki@med.kurume-u.ac.jp )

Kurume University

Yuichi Goto

Kurume University

Shin Sasaki

Kurume University

Shogo Fukutomi

Kurume University

Toru Hisaka

Kurume University

Fumihiko Fujita

Kurume University

Yoshito Akagi

Kurume University

Koji Okuda

Kurume University

\section{Research Article}

Keywords: GNRI, hepatocellular, RFS

Posted Date: February 19th, 2021

DOI: https://doi.org/10.21203/rs.3.rs-209221/v1

License: (1) (1) This work is licensed under a Creative Commons Attribution 4.0 International License.

Read Full License

Version of Record: A version of this preprint was published at Scientific Reports on April 27th, 2021. See the published version at https://doi.org/10.1038/s41598-021-88254-z. 


\section{Abstract}

The geriatric nutritional risk index (GNRI) is widely used for nutritional assessment in older inpatients and was recently reported to be associated with postoperative complications and cancer prognosis. We investigated the use of the GNRI to predict long-term outcomes in hepatocellular carcinoma of all etiologies after hepatectomy. 358 patients were reviewed after propensity score matching. We dichotomized the GNRI score into high GNRI (>98: $N=179)$ and low GNRI ( $\leq 98: N=179)$ and evaluated recurrence-free survival (RFS) and overall survival (OS) between the two groups. Clinicopathological characteristics between the low- and high-GNRI groups were similar after propensity score matching except for the components of the GNRI score (body mass index and serum albumin level), Child-Pugh score (consisting serum albumin level), and preoperative alpha-fetoprotein level $(p<.0001, p<.0001, p=$ 0.0060 , and $p=0.0049$, respectively). A high GNRI was associated with significantly better RFS and OS ( $p$ $=0.0001$ and $p=0.0055$, respectively; log-rank test). Multivariate analysis revealed that GNRI is an independent prognostic factor of RFS and OS (low vs. high; HR, 1.8670; 95\% Cl, 1.4011-2.4880; $p<.0001$, $\mathrm{HR}, 1.7270 ; 95 \% \mathrm{Cl}, 1.1640-2.5623 ; \mathrm{p}=0.0066$, respectively). The GNRI is an objective, inexpensive, and easily calculated assessment tool for nutritional status and can predict prognosis in hepatocellular carcinoma after hepatectomy.

\section{Introduction}

Hepatocellular carcinoma (HCC) is one of the leading causes of cancer-related deaths worldwide ${ }^{1}$. Despite improvements in therapeutic options such as surgery, ablation, trans-arterial chemoembolization, and molecular targeted agents, the outcome of $\mathrm{HCC}$ is unsatisfactory owing to the high recurrence rate. Chronic hepatitis or liver cirrhosis is usually present in the background of HCC and may result in malnutrition in these patients. Some studies have reported that malnutrition negatively impacts postoperative complications and cancer prognosis².

Several nutritional indices are currently in use: the neutrophil/lymphocyte ratio, platelet/lymphocyte ratio, the modified Glasgow prognostic score, the prognostic nutritional index, the controlling nutritional status score, sarcopenia, and the geriatric nutritional index (GNRI). Some researchers have suggested that these indices can predict not only postoperative complications but also the long-term outcomes of several cancers $^{3-5}$. Therefore, these nutritional scores are also considered to be useful for evaluating the postoperative complications and prognosis of $\mathrm{HCC}^{6-8}$.

The GNRI was proposed by Bouillanne et al. in $2005^{9}$ and has been widely used for nutritional assessment in older inpatients with cardiovascular diseases, chronic renal failure with hemodialysis, and chronic liver disease ${ }^{10-13}$. The GNRI is calculated using serum albumin concentration, body height, and body weight, and patients are classified into four nutritional risk grades. Recently, some researchers have reported that the GNRI is associated with postoperative complications and cancer prognosis ${ }^{14,15}$. However, only one report has described this relationship in HCC, and this study was limited to patients 
aged 65 years or older who were positive for the hepatitis B virus ${ }^{16}$. However, the median age of onset of $\mathrm{HCC}$ is around 65 years old, and other underlying liver diseases such as hepatitis virus $\mathrm{C}$, alcoholic and non-alcoholic steatohepatitis are the major causes of HCC in the developed countries. Therefore, we investigated the use of the GNRI to predict long-term outcomes in HCC patients, including those younger than 65 years of age with all underlying liver diseases.

\section{Materials And Methods}

\section{Patients}

Six hundred and forty-eight consecutive patients who underwent hepatectomy for initial HCC at Kurume University between 2008 and 2018 were retrospectively reviewed. Of these, 120 were excluded due to the following reasons: 107 underwent preoperative treatment such as transcatheter arterial infusion, transarterial chemoembolization, and/or ablation; six did not have histologically confirmed HCC (HCC + combined hepatocellular-cholangiocellular carcinoma, necrosis, etc.); three had insufficient data; two did not achieve curative resection; one had another synchronous malignancy; and one had inferior vena cava tumor thrombus. Therefore, 528 patients were finally enrolled for analyses before propensity score matching (PSM). This study was approved by the Research Ethics Committee of Kurume University (No. 20236) and was conducted according to the tenets of the Declaration of Helsinki. The need for informed consent was waived owing to the retrospective nature of the study.

\section{Data collection and calculation of the GNRI scores}

Clinical and pathological data were collected from the patients' medical records. Blood samples and physical data were obtained within 1 week before surgery. Calculation of the GNRI score was as follow: GNRI $=(1.489 \times$ serum albumin concentration $[\mathrm{g} / \mathrm{L}])+(41.7 \times$ present/ideal body weight $[\mathrm{kg}])$. When the present body weight exceeded the ideal body weight, "present/ideal body weight" was set to 1 . Pathological diagnosis was performed by two pathologists in accordance with the rules of the Liver Cancer Study Group of Japan.

\section{Cutoff value of the GNRI score}

The GNRI was categorized into four risk groups: no risk (GNRI: >98), low risk (GNRI: 92 to 98), moderate risk (GNRI: 82 to <92), and major risk (GNRI: <82). We set the cutoff value to $\leq 98$ or $>98$, which was in line with other studies ${ }^{14,17}$. The distribution of the GNRI score in the present study is shown in Supplementary Figure S1.

\section{Surgical procedure}

The type of surgery was comprehensively planned according to the Child-Pugh score, liver damage, platelet count, liver stiffness, the extent of cirrhosis, the presence of esophageal varix and splenomegaly, and patients' comorbidities. Major hepatectomy was defined as lobectomy or more, and minor hepatectomy was defined as sectionectomy or less. Surgical procedures were performed as follows. Liver 
mobilization was performed in most cases, if necessary. The Pringle maneuver was used in most cases according to the surgeon's preference. Parenchymal transection was carried out using an ultrasonic coagulation dissector and/or clamp crushing methods. Sizable vasculature was ligated tightly.

\section{Postoperative follow-up}

In the postoperative follow-up, patients were examined using ultrasonography and tumor markers (alphafetoprotein [AFP] and/or protein induced by vitamin $\mathrm{K}$ absence or antagonist-II [PIVKA-II]) at least every 3 months after surgery. Additionally, computed tomography (CT) and/or magnetic resonance imaging (MRI) were performed at least every 6 months in the patients with a high risk of recurrence. If recurrence was suspected, further examinations such as gadolinium ethoxybenzyl diethylenetriamine pentaacetic acid-enhanced MRI or contrast-enhanced ultrasonography were conducted. Recurrence-free survival (RFS) was defined as the time from surgery to recurrence or death. Overall survival (OS) was defined as the time from surgery to death.

\section{Statistical analyses}

The clinicopathological characteristics were compared using the chi-square test for categorical variables and the Mann-Whitney U test for continuous variables. PSM was performed to overcome selection bias. The propensity score was calculated using a logistic regression model. Covariates entered into the PSM model were as follow: age, sex, total bilirubin level, prothrombin time, platelet count, AFP level, duration of surgery, estimated blood loss, surgical procedure, tumor diameter, tumor number, tumor differentiation, microvascular invasion (MVI), and histological fibrosis grade. PSM was performed using a 1:1 matching method with a caliper width of 0.2 , and the area under the curve calculated from the receiver operating characteristics curve was $0.68703(p<.0001)$. Survival curves were created using the Kaplan-Meier method and compared using the log-rank test. A Cox proportional hazards model was used for univariate and multivariate analyses, and hazard ratios (HR) and 95\% confidence intervals ( $\mathrm{Cl})$ were calculated. All statistical analyses were performed using JMP Pro, version 15 (SAS Institute, Cary, NC). A p-value $<0.05$ was considered statistically significant.

\section{Results}

\section{Patient characteristics}

Clinicopathological features between the low- and high-GNRI groups in the entire cohort before PSM are summarized in Supplementary Table S1. Age, body mass index (BMI), total bilirubin level, serum albumin concentrations, prothrombin time, AFP, Child-Pugh score, duration of surgery, and tumor number were significantly different between the two groups $(p=0.0002, p<.0001, p=0.015, p<.0001, p=0.0005, p=$ $0.0001, p=0.0004, p=0.0126$, and $p=0.0286$, respectively). After matching, almost all variables were adjusted except for the components of the GNRI score (BMI and serum albumin level), Child-Pugh score (consisting serum albumin level), and preoperative AFP level $(p<.0001, p<.0001, p=0.0060$, and $p=$ 0.0049 , respectively) (Table 1 ). 


\section{Comparison of survival between the low- and high-GNRI groups}

The RFS and OS curves of the two groups are shown in Fig. 1. A high GNRI was associated with significantly better RFS and OS ( $p=0.0001$ and $p=0.0055$, respectively).

\section{Univariate and multivariate analyses for RFS and OS}

The results of the univariate and multivariate analyses of RFS and OS are shown in Tables 2 and 3. Univariate analysis of RFS demonstrated that underlying liver disease (Viral vs. Non viral; HR, 1.4695; 95\% $\mathrm{Cl}, 1.0522-2.0524 ; \mathrm{p}=0.0239)$, AFP level ( $5>\mathrm{vs}$. $\geq 5 \mathrm{ng} / \mathrm{mL} ; \mathrm{HR}, 0.6145 ; 95 \% \mathrm{Cl}, 0.4386-0.8608 ; \mathrm{p}=$ $0.0046)$, tumor diameter ( $20 \geq$ vs. $>20 \mathrm{~mm} ; \mathrm{HR}, 0.6152 ; 95 \% \mathrm{Cl}, 0.4520-0.8374 ; \mathrm{p}=0.0020)$, tumor number (solitary vs. multiple; $\mathrm{HR}, 0.6341 ; 95 \% \mathrm{Cl}, 0.4639-0.8668 ; \mathrm{p}=0.0043$ ), tumor differentiation (well/mod vs. poor; HR, 0.6337; 95\% Cl, 0.4506-0.8912; $\mathrm{p}=0.0087$ ), MVI (-vs. +; HR, 0.5300; 95\% Cl, $0.3970-0.7074 ; p<.0001$ ), and GNRI (low vs. high; HR, 1.7193; 95\% Cl, 1.3000-2.2737; $\mathrm{p}=0.0001$ ) were the prognostic factors. On multivariate analysis, underlying liver disease (Viral vs. Non viral; HR, 1.5964; $95 \% \mathrm{Cl}, 1.1296-2.2562 ; \mathrm{p}=0.0080)$, tumor diameter $(20 \geq \mathrm{vs} .>20 \mathrm{~mm} ; \mathrm{HR}, 0.6385 ; 95 \% \mathrm{Cl}, 0.4618-$ $0.8829 ; p=0.0067$ ), tumor number (solitary vs. multiple; $\mathrm{HR}, 0.6046 ; 95 \% \mathrm{Cl}, 0.4383-0.8339 ; \mathrm{p}=0.0022$ ), MVI (- vs. +; HR, 0.5940; 95\% Cl, 0.4346-0.8118; $\mathrm{p}=0.0011$ ), and GNRI (low vs. high; HR, 1.8670; 95\% Cl, $1.4011-2.4880 ; \mathrm{p}<.0001)$ were the independent prognostic factors. Univariate analysis of OS showed that estimated blood loss ( $500>v s$. $\geq 500 \mathrm{~mL} ; \mathrm{HR}, 0.6832 ; 95 \% \mathrm{Cl}, 0.4670-0.9996 ; p=0.0498$ ), tumor diameter ( $20 \geq$ vs. $>20 \mathrm{~mm}$; HR, $0.6186 ; 95 \% \mathrm{Cl}, 0.3996-0.9576 ; p=0.0312)$, tumor differentiation (well/mod vs. poor; HR, 0.5217; $95 \% \mathrm{Cl}, 0.3372-0.8073 ; \mathrm{p}=0.0035)$, MVI (-vs. +; HR, 0.5175; $95 \% \mathrm{Cl}$, $0.3444-0.7775 ; p=0.0015$ ), and GNRI (low vs. high; HR, 1.7343; 95\% Cl, 1.1698-2.5712; $\mathrm{p}=0.0061$ ) were the prognostic factors. On multivariate analysis, MVI (- vs. +; HR, 0.6180; 95\% Cl, 0.3989-0.9575; $\mathrm{p}=$ 0.0312 ), and GNRI (low vs. high; HR, 1.7270; 95\% Cl, 1.1640-2.5623; $p=0.0066$ ) were the independent prognostic factors.

\section{Discussion}

In the present study, we evaluated the relationship between the GNRI score and the long-term outcomes of HCC after hepatectomy. In the entire cohort, the clinicopathological characteristics were different between the two groups; therefore, PSM was applied to overcome that bias. After PSM, the high-GNRI group demonstrated significantly better RFS and OS according to the log-rank test, and a high GNRI was an independent prognostic factor of better RFS and OS in multivariate analyses. Thus far, only one study has mentioned the relationship between GNRI and the prognosis of HCC. Li et al. reported that a low GNRI resulted in a worse prognosis in HCC; however, their study was limited to hepatitis B virus-associated HCC and patients aged 65 years or older ${ }^{16}$. To the best of our knowledge, this is the first study that evaluated the relationship between GNRI and the long-term outcomes of HCC to include patients younger than 65 years and whole underlying liver disease in HCC. 
Many studies have described the usefulness of sarcopenia with respect to postoperative complications and cancer prognosis ${ }^{18,19}$. Although sarcopenia is a powerful tool to evaluate nutritional status, the measurements are complex. Measurements of calf circumference, 6-meter walking time, handgrip strength, 5-times sit-to-standing time, and/or the area of the iliopsoas muscle at the third lumbar vertebra level on CT, etc., are required. On the other hand, the GNRI is an objective, inexpensive, and readily available assessment tool of nutritional status. Only serum albumin level, body height, and weight are needed to calculate the GNRI. Shoji et al. reported a correlation between the area of the iliopsoas muscle at the third lumbar vertebra level and the GNRI score ${ }^{20}$. Besides, Cereda and Vanotti indicated that the GNRI was associated with mid-upper arm muscle circumference, arm muscle area, handgrip strength, and handgrip strength/arm muscle area ${ }^{21}$. Therefore, the GNRI may be a useful tool for predicting postoperative complications and prognosis, especially in patients in whom evaluating sarcopenia is challenging and in institutes that lack the appropriate measuring equipment.

Serum albumin concentration is the main screening tool for immune nutritional status. Hypoalbuminemia induces an impaired immune response, and immunity has a strong influence on cancer prognosis 22,23 . Additionally, a low albumin level is associated with elevated inflammatory cytokines such as tumor necrosis factor-alpha, interleukin-1, and interleukin-6, which may lead to the progression of $\mathrm{HCC}^{24,25}$. Therefore, a low GNRI may reflect impaired tumor immunity which may cause cancer progression.

$\mathrm{BMI}$ is also an immune nutritional index, and several recent studies have mentioned an association between $\mathrm{BMI}$ and the response to immune checkpoint inhibitors ${ }^{26,27}$. A high BMI was reported to be associated with improved survival in patients treated with immune checkpoint inhibitors. Cortellini et al. reported that adipose tissue could activate cytotoxic T-cells and decrease regulatory T-cells. Thus, BMI might influence host immunity. The GNRI is based on serum albumin concentration and BMI, and high values for these components can positively influence host immunity which may improve the cancer prognosis.

Our results indicated that preoperative malnutrition causes worse outcomes after hepatectomy in HCC. Interventions aimed at preoperative nutritional status will improve not only short-term outcomes but also cancer prognosis. Several studies have demonstrated that perioperative nutritional support improves morbidity and prognosis ${ }^{28-30}$. Therefore, we believe that perioperative immune nutritional support should be performed intensively for patients who are planned to undergo hepatectomy.

The present study has some study limitations. First, there is a potential risk of selection bias owing to the single-center, retrospective design. Prospective, multi-institutional studies are needed to validate our results. Second, postoperative complications were not assessed in the present study.

In conclusion, the GNRI is an objective, inexpensive, and easily calculated assessment tool for nutritional status. Our findings suggest that GNRI can be a useful predictor of survival in HCC after hepatectomy. Perioperative nutritional support might improve cancer survival. 


\section{Declarations}

Acknowledgements: We thank Editage for editing the manuscript (https://www.editage.com).

\section{Author's contribution}

K.H. designed the study and developed the methodology. K.H., G.Y., S.S., and F.S. collected the data. K.H. analyzed and interpreted the data. K. H. and O.K. wrote and reviewed the main manuscript text. F.F., H.T., A.Y., and O.K. supervised the study.

\section{Disclosure statements}

Ethics approval: This study was approved by the Research Ethics Committee of Kurume University (Approval number: No. 20236) and was conducted in accordance with the Declaration of Helsinki. The need for informed consent was waived owing to the retrospective nature of the study.

Conflict of Interests: None declared.

Funding: None declared.

\section{References}

1. Siegel, R. L., Miller, K. D. \& Jemal, A. Cancer statistics, 2019. CA Cancer J. Clin.69, 7-34 (2019).

2. Kawaguchi, T. et al. Changes in prognostic factors for patients with hepatocellular carcinoma underwent transarterial chemoembolization with the transition of the time: Child-Pugh class, Albumin-Bilirubin grade, and then. Ann. Trans/ Med.8, 1045 https://doi.org/10.21037/atm-2020-112 (2020).

3. Sasaki, H., Nagano, S., Komiya, S., Taniguchi, N. \& Setoguchi, T. Validation of different nutritional assessment tools in predicting prognosis of patients with soft tissue spindle-cell sarcomas. Nutrients.10, 765 https://doi.org/10.3390/nu10060765 (2018).

4. Matsukawa, T. et al. Validation and comparison of prognostic values of GNRI, PNI, and CONUT in newly diagnosed diffuse large B cell lymphoma. Ann. Hematol.99, 2859-2868 (2020).

5. Takamizawa, Y. et al. Nutritional and inflammatory measures predict survival of patients with stage IV colorectal cancer. BMC Cancer.20, 1092 https://doi.org/10.1186/s12885-020-07560-3 (2020).

6. Harimoto, N. et al. Prognostic Significance of Preoperative Controlling Nutritional Status (CONUT) Score in Patients Undergoing Hepatic Resection for Hepatocellular Carcinoma: A Multi-institutional Study. Ann. Surg Oncol.25, 3316-3323 (2018).

7. Li, L. et al. Early postoperative controlling nutritional status (CONUT) score is associated with complication III-V after hepatectomy in hepatocellular carcinoma: A retrospective cohort study of 1,334 patients. Sci Rep.8, 13406 https://doi.org/10.1038/s41598-018-31714-w (2018). 
8. Imai, D. et al. Prognostic nutritional index is superior as a predictor of prognosis among various inflammation-based prognostic scores in patients with hepatocellular carcinoma after curative resection. Hepatol Res.50, 101-109 (2020).

9. Bouillanne, O. et al. Geriatric Nutritional Risk Index: A new index for evaluating at-risk elderly medical patients. Am J. Clin Nutr.82, 777-783 (2005).

10. Narumi, T. et al. The prognostic importance of objective nutritional indexes in patients with chronic heart failure. J. Cardiol.62, 307-313 (2013).

11. Kinugasa, Y. et al. Geriatric nutritional risk index predicts functional dependency and mortality in patients with heart failure with preserved ejection fraction. Circ J.77, 705-711 (2013).

12. Yamada, K. et al. Simplified nutritional screening tools for patients on maintenance hemodialysis. Am J. Clin Nutr.87, 106-113 (2008).

13. Taniguchi, E. et al. Nutritional assessments for ordinary medical care in patients with chronic liver disease. Hepatol Res.43, 192-199 (2013).

14. Sasaki, M. et al. The Geriatric Nutritional Risk Index predicts postoperative complications and prognosis in elderly patients with colorectal cancer after curative surgery. Sci Rep.10, 10744 https://doi.org/10.1038/s41598-020-67285-y (2020).

15. Kim, Y. N. et al. Comparison of Postoperative Nutritional Status after Distal Gastrectomy for Gastric Cancer Using Three Reconstructive Methods: a Multicenter Study of over 1300 Patients. J. Gastrointest Surg.24, 1482-1488 (2020).

16. Li, L. et al. Geriatric nutritional risk index predicts prognosis after hepatectomy in elderly patients with hepatitis B virus-related hepatocellular carcinoma. Sci Rep.8, 12561 https://doi.org/10.1038/s41598018-30906-8 (2018).

17. Miyake, H., Tei, H. \& Fujisawa, M. Geriatric Nutrition Risk Index is an Important Predictor of CancerSpecific Survival, but not Recurrence-Free Survival, in Patients Undergoing Surgical Resection for Non-Metastatic Renal Cell Carcinoma. Curr Urol.10, 26-31 (2017).

18. Tan, B. H., Birdsell, L. A., Martin, L., Baracos, V. E. \& Fearon, K. C. H. Sarcopenia in an overweight or obese patient is an adverse prognostic factor in pancreatic cancer. Clin Cancer Res.15, 6973-6979 (2009).

19. Harimoto, N. et al. Sarcopenia as a predictor of prognosis in patients following hepatectomy for hepatocellular carcinoma. Br J. Surg.100, 1523-1530 (2013).

20. Shoji, F. et al. Relationship between preoperative sarcopenia status and immuno-nutritional parameters in patients with early-stage non-small cell lung cancer. Anticancer Res.37, 6997-7003 (2017).

21. Cereda, E. \& Vanotti, A. The new Geriatric Nutritional Risk Index is a good predictor of muscle dysfunction in institutionalized older patients. Clin Nutr.26, 78-83 (2007).

22. Galon, J. et al. Type, density, and location of immune cells within human colorectal tumors predict clinical outcome. Science.313, 1960-1964 (2006). 
23. Kanno, H. et al. Differences in the immunosurveillance pattern associated with DNA mismatch repair status between right-sided and left-sided colorectal cancer. Cancer Sci.111, 3032-3044 (2020).

24. Yeh, S. S. \& Schuster, M. W. Geriatric cachexia: The role of cytokines. Am J. Clin Nutr.70, 183-197 (1999).

25. Kotler, D. P. Cachexia - Review. Ann. Intern Med.133, 622-634 (2000).

26. Ichihara, E. et al. The impact of body mass index on the efficacy of anti-PD-1/PD-L1 antibodies in patients with non-small cell lung cancer. Lung Cancer.139, 140-145 (2020).

27. Cortellini, A. et al. A multicenter study of body mass index in cancer patients treated with anti-PD1/PD-L1 immune checkpoint inhibitors: when overweight becomes favorable. J. Immunother Cancer.7, 57 https://doi.org/10.1186/s40425-019-0527-y (2019).

28. Zhao, Y., Qin, H., Wu, Y. \& Xiang, B. Enhanced recovery after surgery program reduces length of hospital stay and complications in liver resection: A PRISMA-compliant systematic review and metaanalysis of randomized controlled trials. Medicine (Baltimore).96, e7628 https://doi.org/10.1097/MD.0000000000007628 (2017).

29. Okabayashi, T. et al. Effects of branched-chain amino acids-enriched nutrient support for patients undergoing liver resection for hepatocellular carcinoma. J. Gastroenterol Hepatol.23, 1869-1873 (2008).

30. Gustafsson, U. O., Oppelstrup, H. \& Thorell, A. Adherence to the ERAS protocol is associated with 5year survival after colorectal cancer surgery: a retrospective cohort study. World J. Surg.40, 17411747 (2016).

\section{Tables}

Due to technical limitations, table 1,2,3 is only available as a download in the Supplemental Files section.

\section{Figures}




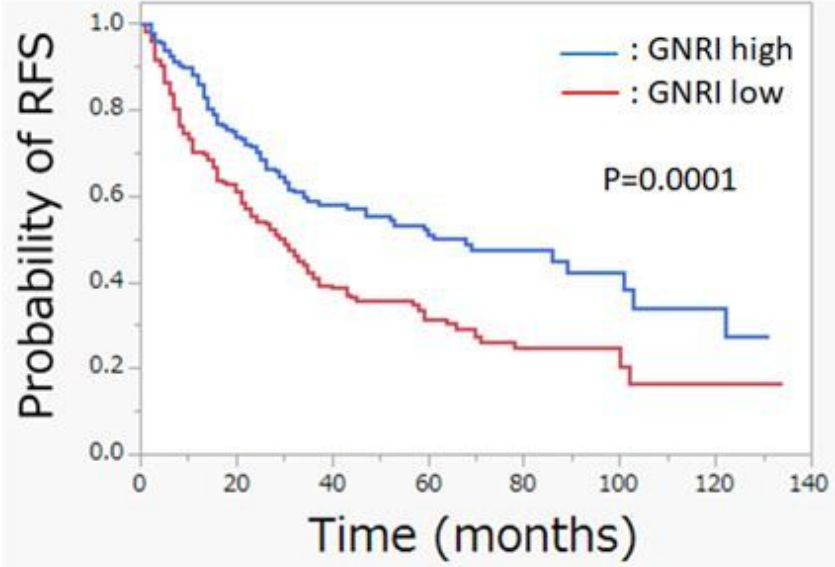

No. at risk

$\begin{array}{llllllll}\text { GNRI high } & 179 & 128 & 75 & 46 & 26 & 13 & 6\end{array}$

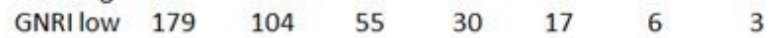

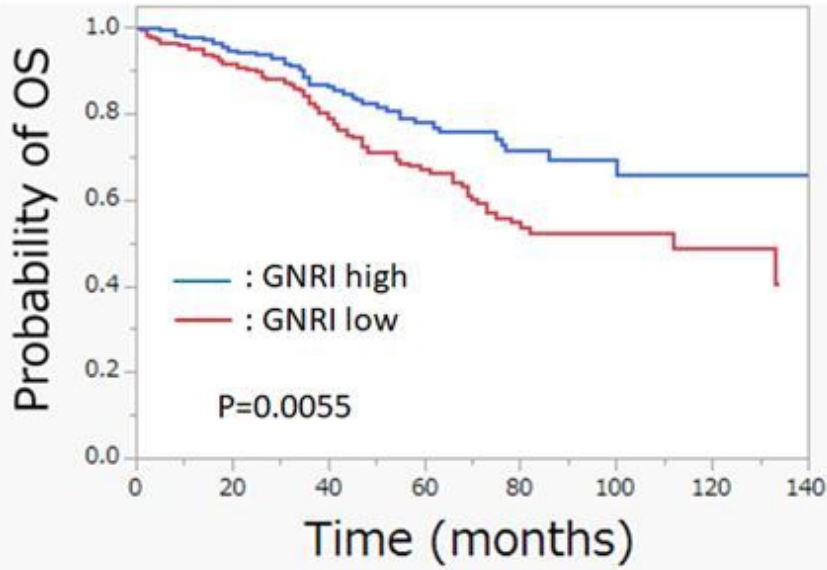

No. at risk

$\begin{array}{llllllll}\text { GNRI high } & 179 & 166 & 120 & 79 & 44 & 20 & 12\end{array}$

$\begin{array}{llllllll}\text { GNRI low } & 179 & 162 & 124 & 77 & 46 & 22 & 14\end{array}$

\section{Figure 1}

Kaplan-Meier curves of RFS and OS in the low- and high-GNRI groups RFS, recurrence-free survival; OS, overall survival, GNRI, geriatric nutritional risk index

\section{Supplementary Files}

This is a list of supplementary files associated with this preprint. Click to download.

- SpplementaryFiles.pdf

- Table1.jpg

- Table2.jpg

- Table3.jpg 